\title{
Haloperidol and delirium in the ICU: the finger pointing to the moon
}

\author{
Nicola Latronico ${ }^{1,2^{*}}$ (1)
}

(0) 2018 Springer-Verlag GmbH Germany, part of Springer Nature and ESICM

Haloperidol, the neuroleptic agent par excellence, was synthesized 60 years ago in February 1958. Since then it has been used in hundreds of thousands of patients with schizophrenia and other psychoses, particularly for the management of psychosis-induced agitation [1], and is included in the World Health Organisation's list of essential medicines.

In 1974-1975, Seeman, by using a preparation of rat brain striatum, discovered that haloperidol selectively blocked the D2 dopamine receptors [2]. This research laid the foundations of the dopamine hypothesis of schizophrenia, according to which meso-cortico-limbic dopamine pathways overactivation has a central role in this disease. This hypothesis also provides a biological basis to explain the observed efficacy of haloperidol not only in schizophrenia but also in delirium. Dopamine excess may cause some of the neurobehavioral alterations observed in patients with hyperactive or mixed type delirium, namely agitation, restlessness, irritability, increased psychomotor activity, distractibility, hyperalertness, combativeness, and psychotic distressing symptoms [3]. This explains why dopaminergic drugs, such as the levodopa, can precipitate delirium, while dopamine antagonists like haloperidol and other antipsychotics can effectively control the behavioral signs of delirium. Dopamine D2 antagonists enhance acetylcholine release, which may be another mechanism by which these drugs help to alleviate the symptoms of delirium [3]. Based on this multiplicity of effects, some experts suggest that haloperidol and other antipsychotic agents may be effective not only in the management of behavioral symptoms of delirium

\footnotetext{
*Correspondence: nicola.latronico@unibs.it

2 Department of Anesthesia, Critical Care and Emergency, Spedali Civili University Hospital, Piazzale Ospedali Civili, 1, 25123 Brescia, Italy

Full author information is available at the end of the article
}

(agitation), but they might also be useful in patients with hypoactive delirium to control distressing psychotic symptoms such as hallucinations and delusions [4].

In animals, dopamine agonists cause slowing of EEG despite motor hyperactivity, which matches the features of hyperactive delirium [3]. More recent research has shown that D2 dopamine receptors in the cortex are a common target for both typical (haloperidol) and atypical antipsychotics, but these latter induce a significantly lower D2 binding than haloperidol in the basal ganglia, particularly in the striatum [5] (Fig. 1a). This fits well with the association of antipsychotic efficacy (attributed to an anti-dopaminergic effect on the meso-corticolimbic dopamine pathways) and lower extra-pyramidal side effects (attributed to anti-dopaminergic effect in the striatum) in atypical antipsychotics than with haloperidol. Study in human volunteers has confirmed that acute administration of haloperidol causes a state of impaired, though rapidly reversible, motor ability coinciding with diminished grey matter volume and connectivity in the striatum, a brain region that mediates movement [6].

Pathophysiology of delirium is currently incompletely understood, with multiple overlapping and interacting mechanisms [7]. The neuroinflammatory hypothesis proposes that systemic inflammation causes increased amounts of pro-inflammatory cytokines and inflammatory mediators to penetrate into the brain, causing neuronal dysfunction and subsequent neurobehavioral and cognitive symptoms of delirium, either directly or by stimulating the microglia to produce inflammatory mediators [7]. If microglia is already primed by preceding events or concurrent neurodegenerative processes, its overactivation leads to $\mathrm{BBB}$ disruption with further brain penetration of pro-inflammatory cytokines and fluid shift with brain edema that alter synaptic transmission and neural excitability, impair microcirculation and favor uncoupling of oxidative phosphorylation with reduced neuronal

\section{Springer}



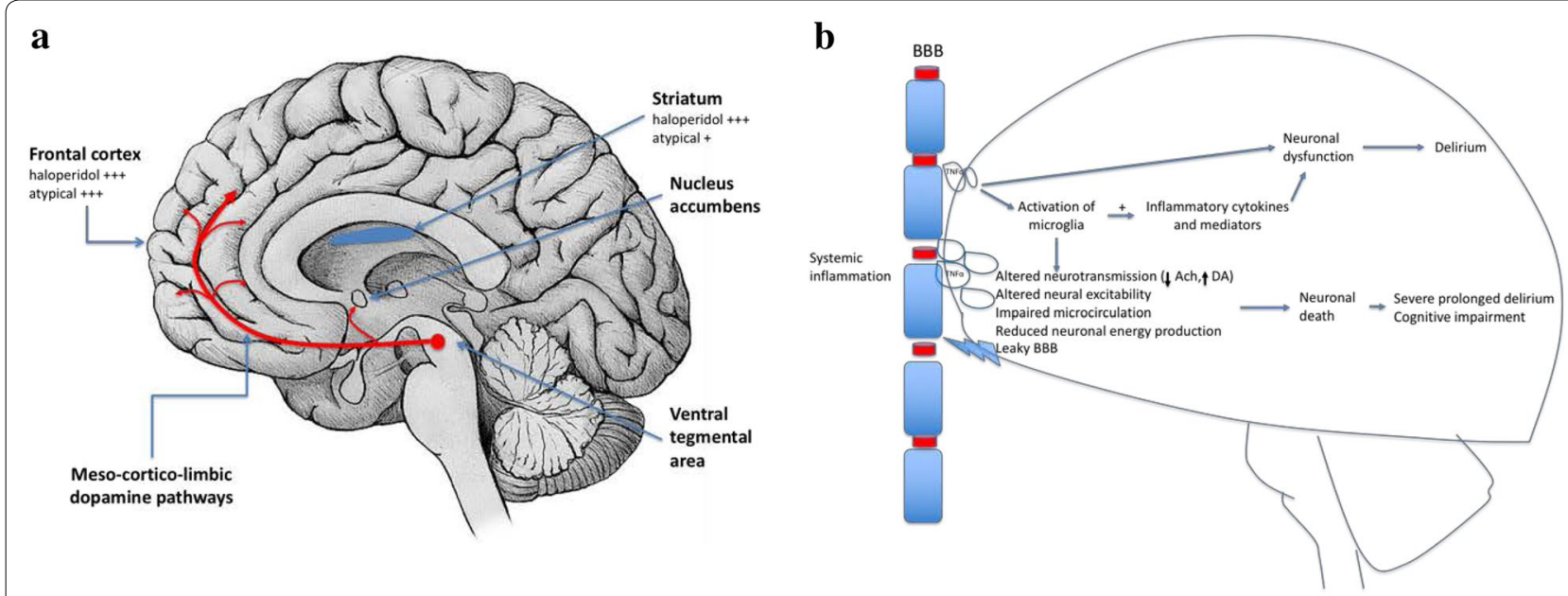

Fig. 1 a Schematic representation of site of action of haloperidol and atypical antipsychotics. The anti-dopaminergic effects are comparable on the meso-cortico-limbic dopamine pathways (responsible for antipsychotic efficacy) but are lower in the striatum (responsible for extra-pyramidal side effects) in atypical antipsychotics than with haloperidol. b Simplified representation of delirium pathogenesis. Brain penetration of TNFa (tumor necrosis factor alfa) together with other inflammatory cytokines (IL-1 beta, IL-6, interferon), chemokines, endothelial adhesion molecules and proteases activates the microglia with further production of inflammatory molecules, blood brain barrier (BBB) leakage and further penetration of inflammatory molecules (TNFa larger drops) causing neuronal dysfunction and death. Brain edema formation alters synaptic transmission, neural excitability and neurotransmitter balance (decreased acetylcholine [Ach] and increased dopamine [DA]), impairs microcirculation and favors ischemia. A global representation of pathogenetic hypotheses of delirium and their interaction is presented in Ref. [7]

energy production and ischemia [7, 8]. This theory has received recent support in ICU patients by research showing that S100B, a marker of BBB and/or astrocyte injury, and E-selectin, an adhesion molecule and marker of endothelial injury, are associated with long-term cognitive impairment after critical illness [9]. Reduced cholinergic activity (and, hence, reduced inhibition of microglia activity), increased release of norepinephrine, glutamate and dopamine, as well as the imbalance of other neurotransmitters create a vicious cycle of neuroinflammation, neurotransmitter imbalance and ischemic damage leading to neuronal death, severe prolonged delirium and long term cognitive impairment (Fig. 1b).

First use of intravenous haloperidol for treating delirium in the ICU dates back to 1980, shortly after the publication of DSM-III defining delirium [10]. Haloperidol has since been the most common drug used to control symptoms of delirium in the ICU patient [10]. This is confirmed in the recent AID-ICU published in Intensive Care Medicine, the largest prospective multinational cohort study published to date on the use of haloperidol in the ICU [11]. The study took place in 99 ICUs from 13 countries and enrolled 1260, of whom 314 patients (25\%) developed delirium-either hyperactive (6.1\%), hypoactive (5.7\%) or mixed (13.1\%) - a median of 3 days after ICU admission. Of these patients, 145 (46.2\%) were treated with haloperidol. Of note, benzodiazepines were the most common other agent use to treat delirium (36\%). Benzodiazepines, especially when used with continuous infusion, may increase the risk of delirium, although sedation depth and the amount of drug used can be a greater risk factor than the type of sedative used [12]. Considering the entire population, patients receiving haloperidol were 166 (13.2\%), they were more often males, slightly older and more severely ill; they had more often metastatic cancer and sepsis at admission, and had received more often haloperidol and benzodiazepine before ICU admission.

Interestingly, haloperidol was administered regardless of delirium subtype and, in a significant proportion of patients, at fixed dose. This indicates a divergence between daily practice and suggested recommendations. Haloperidol is indicated in hyperactive (agitated) delirium, not in hypoactive delirium. Hallucinations and delusions can be an indication to use haloperidol even in hypoactive delirium, but they are exceedingly difficult to assess in the ICU patient. Moreover, haloperidol should be administered until agitation is controlled and no longer thereafter [13]. At fixed doses, the use of haloperidol (and of any other antipsychotic medication) would be prophylactic, which is not currently recommended [14]. A fixed dose regimen may increase the total dose of haloperidol and the risk of side effects, such as extra-pyramidal and cardiac effects. These operational aspects should be clarified in future randomized controlled clinical trials.

Haloperidol use was not associated with increased mortality but the study was underpowered to detect a 90-day mortality difference. Moreover, studying the 
association between delirium and mortality might be of greater clinical importance, particularly if the duration and severity of delirium are taken into consideration, because the negative impact on outcome is mainly limited to patients with persisting delirium [15]. Future studies aiming at assessing haloperidol safety and efficacy, should consider this goal in the context of a drug that is used to treat a clinically relevant disease condition carrying a high risk of long term mortality and morbidity.

\section{Author details}

${ }^{1}$ Department of Medical and Surgical Specialties, Radiological Sciences and Public Health, University of Brescia, Brescia, Italy. ${ }^{2}$ Department of Anesthesia, Critical Care and Emergency, Spedali Civili University Hospital, Piazzale Ospedali Civili, 1, 25123 Brescia, Italy.

\section{Compliance with ethical standards}

\section{Conflicts of interest}

The authors declare that that there is no conflict of interest.

Received: 3 June 2018 Accepted: 7 June 2018

Published online: 23 June 2018

\section{References}

1. Lopez-Munoz F, Alamo C (2009) The consolidation of neuroleptic therapy: Janssen, the discovery of haloperidol and its introduction into clinical practice. Brain Res Bull 79:130-141

2. Madras BK (2013) History of the discovery of the antipsychotic dopamine D2 receptor: a basis for the dopamine hypothesis of schizophrenia. J Hist Neurosci 22:62-78

3. Maldonado JR (2008) Pathoetiological model of delirium: a comprehensive understanding of the neurobiology of delirium and an evidencebased approach to prevention and treatment. Crit Care Clin 24:789-856

4. Maldonado JR (2013) Neuropathogenesis of delirium: review of current etiologic theories and common pathways. Am J Geriatr Psychiatry 21:1190-1222

5. Xiberas X, Martinot JL, Mallet L, Artiges E, Loc HC, Maziere B, Paillere-Martinot ML (2001) Extrastriatal and striatal D(2) dopamine receptor blockade with haloperidol or new antipsychotic drugs in patients with schizophrenia. Br J Psychiatry 179:503-508
6. Tost H, Braus DF, Hakimi S, Ruf M, Vollmert C, Hohn F, Meyer-Lindenberg A (2010) Acute D2 receptor blockade induces rapid, reversible remodeling in human cortical-striatal circuits. Nat Neurosci 13:920-922

7. Maldonado JR (2017) Delirium pathophysiology: an updated hypothesis of the etiology of acute brain failure. Int J Geriatr Psychiatry. https://doi. org/10.1002/gps.4823

8. van Gool WA, van de Beek D, Eikelenboom P (2010) Systemic infection and delirium: when cytokines and acetylcholine collide. Lancet 375:773-775

9. Hughes CG, Patel MB, Brummel NE, Thompson JL, MCNeil JB, Pandharipande PP, Jackson JC, Chandrasekhar R, Ware LB, Ely EW, Girard TD (2018) Relationships between markers of neurologic and endothelial injury during critical illness and long-term cognitive impairment and disability. Intensive Care Med 44:345-355

10. Sos JC, Cassem NH (1980) The intravenous use of haloperidol for acute delirium in intensive care setting. In: Speidel H, Rodewald G (eds) Psychic and neurological dysfunctions after open heart surgery. Thieme, Stuttgart, pp 196-199

11. Collet MO, Caballero J, Sonneville R, Bozza FA, Nydahl P, Schandl A, Woien H, Citerio G, van den Boogaard M, Hastbacka J, Haenggi M, Colpaert K, Rose L, Barbateskovic M, Lange T, Jensen A, Krog MB, Egerod I, Nibro HL, Wetterslev J, Perner A, the AID-ICU Cohort Study Co-authors (2018) Prevalence and risk factors related to haloperidol use for delirium in adult intensive care patients: the multinational AID-ICU inception cohort study. Intensive Care Med. https://doi.org/10.1007/s00134-018-5204-y

12. Girard TD, Thompson JL, Pandharipande PP, Brummel NE, Jackson JC, Patel MB, Hughes CG, Chandrasekhar R, Pun BT, Boehm LM, Elstad MR, Goodman RB, Bernard GR, Dittus RS, Ely EW (2018) Clinical phenotypes of delirium during critical illness and severity of subsequent long-term cognitive impairment: a prospective cohort study. Lancet Respir Med 6:213-222

13. Salluh JIF; Latronico N, (2018) Does this critically ill patient with delirium require any drug treatment? Intensive Care Med

14. van den Boogaard M, Slooter AJC, Bruggemann RJM, Schoonhoven L, Beishuizen A, Vermeijden JW, Pretorius D, de Koning J, Simons KS, Dennesen PJW, Van der Voort PHJ, Houterman S, van der Hoeven JG, Pickkers $P$, Investigators RS, van der Woude MCE, Besselink A, Hofstra LS, Spronk PE, van den Bergh W, Donker DW, Fuchs M, Karakus A, Koeman M, van Duijnhoven M, Hannink G (2018) Effect of haloperidol on survival among critically ill adults with a high risk of delirium: the REDUCE randomized clinical trial. JAMA 319:680-690

15. Salluh Jl, Wang H, Schneider EB, Nagaraja N, Yenokyan G, Damluji A, Serafim RB, Stevens RD (2015) Outcome of delirium in critically ill patients: systematic review and meta-analysis. BMJ 350:h2538 